\title{
Cerebrovascular Reactivity during Prolonged Breath-Hold in Experienced Freedivers
}

\author{
(D) V.C. Keil, (D) E. Eichhorn, DH.J.M.M. Mutsaerts, (D)F. Träber, (D)W. Block, (D)B. Mädler, (DK. van de Ven, (D).C.W. Siero, \\ (D) B.J. Maclntosh, (D). Petr, (D) R. Fimmers, (D)H.H. Schild, and (D) E. Hattingen
}

\begin{abstract}
BACKGROUND AND PURPOSE: Experienced freedivers can endure prolonged breath-holds despite severe hypoxemia and are therefore ideal subjects to study apnea-induced cerebrovascular reactivity. This multiparametric study investigated CBF, the spatial coefficient of variation as a correlate of arterial transit time and brain metabolism, dynamics during prolonged apnea.
\end{abstract}

MATERIALS AND METHODS: Fifteen male freedivers (age range, 20-64 years; cumulative previous prolonged breath-holds $>2$ minutes and 30 seconds: 4-79,200) underwent repetitive 3T pseudocontinuous arterial spin-labeling and ${ }^{31} \mathrm{P}-/{ }^{1} \mathrm{H}-\mathrm{MR}$ spectroscopy before, during, and after a 5-minute breath-hold (split into early and late phases) and gave temporally matching venous blood gas samples. Correlation of temporal and regional cerebrovascular reactivity to blood gases and cumulative previous breath-holds of $>2$ minutes and 30 seconds in a lifetime was assessed.

RESULTS: The spatial coefficient of variation of CBF (by arterial spin-labeling) decreased during the early breath-hold phase $(-30.0 \%, P=$ .002), whereas CBF remained almost stable during this phase and increased in the late phase $(+51.8 \%, P=.001)$. CBF differed between the anterior and the posterior circulation during all phases (eg, during late breath-hold: MCA, $57.3 \pm 14.2$ versus posterior cerebral artery, $42.7 \pm$ $10.8 \mathrm{~mL} / 100 \mathrm{~g} / \mathrm{min} ; P=.001)$. There was an association between breath-hold experience and lower CBF (1000 previous breath-holds reduced WM CBF by $0.6 \mathrm{~mL} / 100 \mathrm{~g} / \mathrm{min} ; 95 \% \mathrm{Cl}, 0.15-1.1 \mathrm{~mL} / 100 \mathrm{~g} / \mathrm{min} ; P=.01)$. While breath-hold caused peripheral lactate rise (+18.5\%) and hypoxemia (oxygen saturation, $-24.0 \%$ ), cerebral lactate and adenosine diphosphate remained within physiologic ranges despite early signs of oxidative stress [ $-6.4 \%$ phosphocreatine / (adenosine triphosphate + adenosine diphosphate); $P=.02$ ].

CONCLUSIONS: This study revealed that the cerebral energy metabolism of trained freedivers withstands severe hypoxic hypercarbia in prolonged breath-hold due to a complex cerebrovascular hemodynamic response.

ABBREVIATIONS: ATP = adenosine triphosphate; ASL = arterial spin-labeling; ASL-sCoV = spatial coefficient of variation of CBF (by ASL); $A T T=$ arterial transit time; $\mathrm{CVR}=$ cerebrovascular reactivity; $\mathrm{HR}=$ heart rate; $\mathrm{P}_{\mathrm{i}}=$ inorganic phosphates; $\mathrm{PCr}=$ phosphocreatine; $\mathrm{PO}_{2}=$ partial pressure of oxygen; $\mathrm{SpO} \mathrm{O}_{2}=$ oxygen saturation

$\mathbf{F}^{\mathrm{r}}$ reedivers acquire the ability to voluntarily breath-hold for several minutes: One breath-hold can be extended up to the world record of 11 minutes and 30 seconds. ${ }^{1}$ However, animal studies have suggested that threshold times for hypoxia-induced neuronal cell death can be as low as 2 minutes and 30 seconds. $^{2}$ The

Received December 29, 2017; accepted after revision July 19, 2018.

From the Departments of Radiology (V.C.K., F.T., W.B., H.H.S., E.H.) and Anesthesiology and Intensive Care Medicine (L.E.), and Institut für Medizinische Biometrie, Informatik und Epidemiologie (R.F.), University Hospital Bonn, Bonn, Germany; Department of Radiology (H.J.M.M.M.), Academic Medical Center, Amsterdam, the Netherlands; Sunnybrook Research Institute (H.J.M.M.M., B.J.M.), University of Toronto, Toronto, Ontario, Canada; Department of Radiology (H.J.M.M.M., J.C.W.S.), University Medical Center Utrecht, Utrecht, the Netherlands; Philips GmbH (B.M), Bonn, Germany; Philips Healthcare (K.v.d.V.), Best, the Netherlands; Spinoza Centre for Neuroimaging (J.C.W.S.), Amsterdam, the Netherlands; and Helmholtz Center Dresden-Rossendorf, Institute for Radiopharmaceutic Cancer Research (J.P.), PET Center, Dresden-Rossendorf, Germany.

Vera C. Keil, Lars Eichorn, and Henri J.M.M. Mutsaerts contributed equally to this work. regulation and sufficiency of cerebrovascular reactivity (CVR) to prevent ischemic brain damage during prolonged breath-hold are unclear. Such knowledge may hold clues to explain patterns of brain damage in adverse diving outcomes and may provide suggestions for targeted therapies. It can also be useful to deduce CVR in medical conditions of repetitive hypoxemia such as obstructive sleep apnea syndrome. ${ }^{3}$

Previous MR imaging breath-hold experiments involved hy-

Paper previously presented, in part, at: 99th German Röntgen Congress, May 27, 2017 (No. 4735), Leipzig, Germany; and Joint Annual Meeting of the International Society for Magnetic Resonance in Medicine and the European Society for Magnetic Resonance in Medicine and Biology, June 16-21, 2018; Paris, France.

Please address correspondence to Vera Catharina Keil, MD. Department of Radiology, University Hospital Bonn, Sigmund-Freud-Str 25, 53127 Bonn, Germany; e-mail: vera.keil@ukbonn.de

三 Indicates article with supplemental on-line table.

http://dx.doi.org/10.3174/ajnr.A5790 
Anthropometric data of freediver volunteers ${ }^{a}$

\begin{tabular}{|c|c|c|c|c|c|c|}
\hline Participant & $\begin{array}{c}\text { Longest } \\
\text { Breath-Hold } \\
\text { until MRI (min) }\end{array}$ & $\begin{array}{c}\text { Start of } \\
\text { Sport (yr } \\
\text { before MRI) }\end{array}$ & $\begin{array}{c}\text { Estimated } \\
\text { Breath-Holds } \\
>2 \text { min } 30 \text { s per Session }\end{array}$ & $\begin{array}{c}\text { Sessions } \\
\text { per Month }\end{array}$ & $\begin{array}{c}\text { Estimated } \\
\text { Cumulative Lifetime } \\
\text { Breath-Holds }>2 \min 30 \mathrm{~s}\end{array}$ & $\begin{array}{l}\text { Age } \\
\text { (yr) }\end{array}$ \\
\hline 1 & 10.6 & 11.3 & 30 & 0.4 & 1632 & 31 \\
\hline 2 & 5.5 & 1.5 & 7 & 8 & 1008 & 36 \\
\hline 3 & 5.4 & 2.0 & 1 & 1 & 24 & 20 \\
\hline 4 & 5.4 & 11.0 & $\sim 0$ & 8 & 4 & 33 \\
\hline 5 & 5.0 & 2.0 & 0.125 & 8 & 24 & 40 \\
\hline 6 & 5.5 & 10.0 & $\sim 0$ & 4 & 4 & 38 \\
\hline 7 & 5.0 & 8.0 & 5 & 8 & 3840 & 61 \\
\hline 8 & 5.5 & 1.5 & 0.5 & 4 & 36 & 34 \\
\hline 9 & 5.0 & 7.0 & 6 & 16 & 8064 & 47 \\
\hline 10 & 6.2 & 9.0 & 6 & 7 & 4536 & 26 \\
\hline 11 & 5.3 & 27.5 & 15 & 16 & 79,200 & 64 \\
\hline 12 & 6.5 & 10.2 & 12 & 28 & 11,712 & 51 \\
\hline 13 & 5.5 & 11.3 & 10 & 16 & 21,760 & 35 \\
\hline 14 & 5.5 & 1.6 & 18 & 10 & 3420 & 32 \\
\hline 15 & 5.0 & 5.0 & 0.083 & 4 & 20 & 50 \\
\hline Median & 5.5 & 8.0 & 6 & 8 & 2526 & 36.0 \\
\hline $95 \% \mathrm{Cl}$ & $5.3-5.5$ & 2.0-11.0 & $0.14-12.0$ & $4.0-9.96$ & $30-6300$ & $32-50$ \\
\hline Minimum & 5.0 & 1.5 & 0 & 0.41 & 4 & 20 \\
\hline Maximum & 10.6 & 27.5 & 30 & 28 & 79,200 & 64 \\
\hline
\end{tabular}

a Participants were interviewed concerning the duration (start of sport) and intensity (freediving sessions per month) and the estimated frequency of breath-holding for $>2$ minutes and 30 seconds per session. The latter could be near zero in freedivers specializing in short high-frequency breath-holds. Some participants breath-held for $>2$ minutes and 30 seconds only as a qualification for this study; hence, their cumulative lifetime breath-holds of $>2$ minutes and 30 seconds before MRI were 4 , while others very frequently underwent prolonged breath-hold.

percarbic air composition or the use of relatively short breathholds. ${ }^{4}$ There are, however, crucial differences between experimental setups applying altered gas composition and those using voluntary breath-hold. In the latter, the human body can be examined as a closed system of internal metabolic gas exchange, while altered-ventilation gas studies are an "open system" approach with ventilation at variable frequencies as a complicating factor. ${ }^{5}$ Quite different from short breath-holds, prolonged breath-hold for several minutes is furthermore a unique mental challenge demanding an emotional preparation, for which freedivers need to train and which may have an independent effect on CVR. ${ }^{6}$ The putative effect of experience from previous prolonged breath-holds on CBF must be mentioned in this context and has not been explored.

Arterial spin-labeling (ASL) perfusion MR imaging allows an in vivo assessment of absolute $\mathrm{CBF}$ both regionally and on a vessel-selective level. ASL studies already successfully identified chronic CVR alterations due to obstructive sleep apnea as well as acute alterations during very short breath-holds. ${ }^{5,7}$ A corollary ASL measure is the spatial coefficient of variation (ASL-sCoV) of the CBF image itself, which was recently identified as a correlate for arterial transit time (ATT) and is a useful alternative when direct ATT estimations are not feasible. ${ }^{8}$ Thus, ASL seems a promising technique to study CVR in prolonged breath-hold.

Blood gas alterations trigger a cerebrovascular but also a peripheral vascular reaction in the body. During breath-hold, for example, the CBF increases and hence faster $\mathrm{O}_{2}$ supply facilitates the maintenance of an aerobic brain metabolism under hypoxemia-that is, to prevent the depletion of adenosine triphosphate (ATP) and the accumulation of lactate from anaerobic glycolysis. The sufficiency of CVR can be studied indirectly by ${ }^{31} \mathrm{P}-\mathrm{MR}$ spectroscopy, which provides estimates of ATP and related metabolites, ${ }^{9}$ as well as by ${ }^{1} \mathrm{H}$-MR spectroscopy, which may show lactate if the metabolite is elevated beyond physiologic ranges.
The goal of the present study in experienced freedivers was to explore the following: 1) acute CVR during a prolonged breathhold challenge with ASL, and 2) the sufficiency of CVR to maintain aerobic cerebral energy metabolism as measured by ${ }^{31} \mathrm{P}$ - and ${ }^{1} \mathrm{H}-\mathrm{MR}$ spectroscopy. In addition, this study investigated whether pre-existing experience with prolonged breath-holds has an influence on the CVR during the breath-hold experiment.

\section{MATERIALS AND METHODS Participants}

Fifteen experienced male freedivers (median age, 36.0 years; 95\% CI, 32.0-50.0 years) participated (Table). Inclusion criteria were adult age and an ability to breath-hold for $>4$ minutes without stress symptoms (ie, tachycardia, oxygen saturation $\left[\mathrm{SpO}_{2}\right]$ below $60 \%$, delirium). Exclusion criteria were pre-existing cardiac or neurologic disorders and current smoking. The participant's age and cumulative number of breathholds longer than 2 minutes and 30 seconds during the volunteer's lifetime (estimated by interview report) were registered as potential influential factors.

\section{Study Design}

Preparatory evaluations involved questionnaires regarding claustrophobia, noise tolerance. or issues with restraints. All participants were instructed to refrain from meals and caffeine for at least 2 hours before MR imaging to reduce perfusion confounders. ${ }^{10}$ Preparation (eg, meditation, test placement on the MR imaging table) was allowed. MR imaging-electrocardiography and finger oximetry guaranteed continuous monitoring of $\mathrm{SpO}_{2}$ and heart rate (HR). Arterial $\mathrm{CO}_{2}$ measurements were not permitted by the hospital ethics committee, but venous blood gas was repetitively analyzed. Continuity of breath-hold and consciousness were visually monitored (L.E.) to guarantee safety and correct measurements. 
The MR imaging protocol consisted of a 3D T1-weighted acquisition followed by 5 ASL scans. Participants were instructed to breathe with normal frequency to obtain the baseline CBF (baseline phase). Hyperventilation to reduce blood $\mathrm{CO}_{2}$ or increase $\mathrm{O}_{2}$ concentrations was a forbidden confounder. The participants gave an acoustic signal when starting the 5-minute breath-hold, when 2 consecutive ASL scans were acquired (early and late breath-hold phases). After the second breath-hold scan, the participant was instructed to breathe again at normal frequency (approximately 16/min.). Without delay, 2 normal-breathing ASL scans (early and late recovery phases) were obtained. A subset of participants was available for ${ }^{31} \mathrm{P}$ - and ${ }^{1} \mathrm{H}$-MR spectroscopy $(n=$ 11 and 8 participants, respectively) during separate sessions to determine relative brain metabolites and, again, venous blood gas. The study was approved by the University Hospital Bonn, Germany ethics committee. All participants provided written informed consent.

\section{MR Imaging Sequences}

All imaging was performed on a 3T Ingenia MR imaging scanner (Philips Healthcare, Best, the Netherlands). The T1-weighted MPRAGE sequence was $1 \times 1 \times 1 \mathrm{~mm}^{3}$. The 5 identical pseudocontinuous ASL sequences were acquired with a $3 \mathrm{D}$ gradient- and spin-echo readout ( 5 segments; acquisition voxel size, $3.75 \times$ $3.75 \times 6 \mathrm{~mm}^{3}$; FOV, $240 \times 240 \times 96 \mathrm{~mm}^{3}$; TE/TR, 8/4.28 s; labeling duration, $1.8 \mathrm{~s}$; radiofrequency labeling pulse duration/ interval, 0.7/1.41 ms; postlabeling delay, $2 \mathrm{~s} ; 4$ background suppression pulses; scan time, 2 minutes and 30 seconds per scan phase). Each sequence consisted of $2 \mathrm{M} 0$ images and 2 controllabel pairs. A labeling distance of $13 \mathrm{~cm}$ (middle slice of the ASL stack to labeling plane) was chosen.

\section{Image Processing}

Image processing was performed with ExploreASL (www.ExploreASL.com), a toolbox based on SPM12 routines (http:// www.fil.ion.ucl.ac.uk/spm/software/spm12), which was initiated through the European Union-funded European Cooperation in Science and Technology (COST) Action ASL In Dementia (http://s434060124.online.de/aslindementiacms/), aiming at harmonizing ASL image processing for single- and multicenter ASL studies. ${ }^{11}$ Image-processing steps were the following: automated segmentation of 3D T1-weighted images using the Computational Anatomy Toolbox (CAT12 toolbox) rigid-body registration of $\mathrm{CBF}$ to the gray matter partial volume map and spatial normalization into a common space using the Diffeomorphic Anatomical Registration Through Exponentiated Lie Algebra Toolbox (DARTEL, part of SPM). ${ }^{12} \mathrm{M} 0$ images were masked, iteratively smoothed, and extrapolated outside the mask, and CBF was quantified using a single-compartment quantification model. ${ }^{13}$ The ROIs analyzed were the total (cortical) GM and the total white matter. The GM was subsegmented into vascular territories of the anterior, middle, and posterior cerebral arteries. The vascular territories were delineated in the common space on the Montreal Neurological Institute atlas according to Tatu et al. ${ }^{14}$

The ASL-sCoV was defined as the SD of CBF divided by the mean CBF, within the total cortical GM (gray matter probability $>0.7)^{8}$ :

$$
A S L-s C o V_{\mathrm{ROI}}=\frac{\sigma\left(C B F_{\mathrm{GM}}\right)}{\mu\left(C B F_{\mathrm{GM}}\right)} \times 100 \% .
$$

Partial volume effects within the GM and WM ROIs were corrected using the method of Asllani et al. ${ }^{15}$

\section{MR Spectroscopy}

Proton-decoupled ${ }^{31} \mathrm{P}$ spectra were acquired with a dual-tunable ${ }^{31} \mathrm{P} /{ }^{1} \mathrm{H}$ birdcage transmit/receive head coil (1024 data points; 3-kHz sampling; TR, 4 seconds; 4 signal averages) as a continuous time-series with 16 -second duration for each spectrum, comprising a total of 40 scans before, during, and directly after breathhold in a 25-mm-thick axial slice on the level of the basal ganglia. ${ }^{31} \mathrm{P}$ signals were processed by the AMARES algorithm of the JAVA-MRUI software (http://www.jmrui.eu/features/quantitation/) quantifying 15 peaks in each spectrum arising from phosphocreatine $(\mathrm{PCr})$, inorganic phosphates $\left(\mathrm{P}_{\mathrm{i}}\right)$, ATP, and phosphomonoand diesters. ${ }^{16}$ The cerebral $\mathrm{pH}$ was determined from the frequency separation between $\mathrm{P}_{\mathrm{i}}$ and PCr. Five consecutive spectra sets within the ${ }^{31} \mathrm{P}$ time-series were averaged for 2 minutes and 30 seconds, which represent the metabolic baseline, first and second halves of breath-hold, as well as early and late metabolic recovery phases. This step was also to improve the signal-to-noise ratio. Thus, the time course of $\mathrm{pH}$ and of the ratios $\mathrm{P}_{\mathrm{i}} / \mathrm{PCr}, \mathrm{P}_{\mathrm{i}} / \beta$-ATP, $\mathrm{PCr} / \beta$-ATP, as well as the ratios of all ${ }^{31} \mathrm{P}$ metabolites relative to (ATP + adenosine diphosphate), calculated from the mean of the $\gamma$ - and $\alpha$-ATP peaks, were obtained in the same 2 minutes and 30 seconds intervals as the ASL sequences. $\gamma$ - and $\alpha$-ATP peaks include the signals from nucleotide diphosphates and thus are expected as near-constant.

In a consecutive single-volume ${ }^{1} \mathrm{H}-\mathrm{MR}$ spectroscopy breathhold experiment, ratios of the ${ }^{1} \mathrm{H}$-MR signals of $N$-acetylaspartate, total creatine, choline, and lactate were determined from an 8 -mL volume in the left basal ganglia (point-resolved spectroscopy sequence localized spectra: TR $2 \mathrm{~s}$; TE $0.14 \mathrm{~s} ; 128$ signal averages). Recording periods were split to match the ASL-MR imaging and ${ }^{31} \mathrm{P}-\mathrm{MR}$ spectroscopy phases of the breath-hold experiment.

\section{Blood Gas Analysis}

We drew $10 \mathrm{~mL}$ of venous blood before breath-hold, after $2 \mathrm{~min}$ utes and 30 seconds of breath-hold, at first breath after breathhold, and after 2 minutes and 30 seconds of recovery. Immediate analysis (RAPIDLab 1265; Siemens, Erlangen, Germany) involved partial pressure of $\mathrm{CO}_{2}$ and oxygen $\left(\mathrm{pO}_{2}\right)$, glucose, and lactate levels. With the exception of partial pressure of $\mathrm{CO}_{2}$, these parameters were shown to correlate well with arterial values. ${ }^{17}$

\section{Statistics}

Overall CBF variability was analyzed by a mixed linear model with the participant as a random factor, while differences between single time points were analyzed by a paired $t$ test, which considers $\mathrm{CBF}$ differences compared with baseline $\left(\Delta \mathrm{CBF} / \mathrm{CBF}_{0}\right)$ as the expression of CVR without division by a fixed rate of stimulus such as per unit $\mathrm{CO}_{2} \cdot{ }^{18}$ The same tests were applied for the partial volume-corrected ASL-sCoV. MR spectroscopy parameters were analyzed in a mixed linear model and with the Pearson $R$ correlation. The GM CBF in the vascular territories was analyzed sepa- 


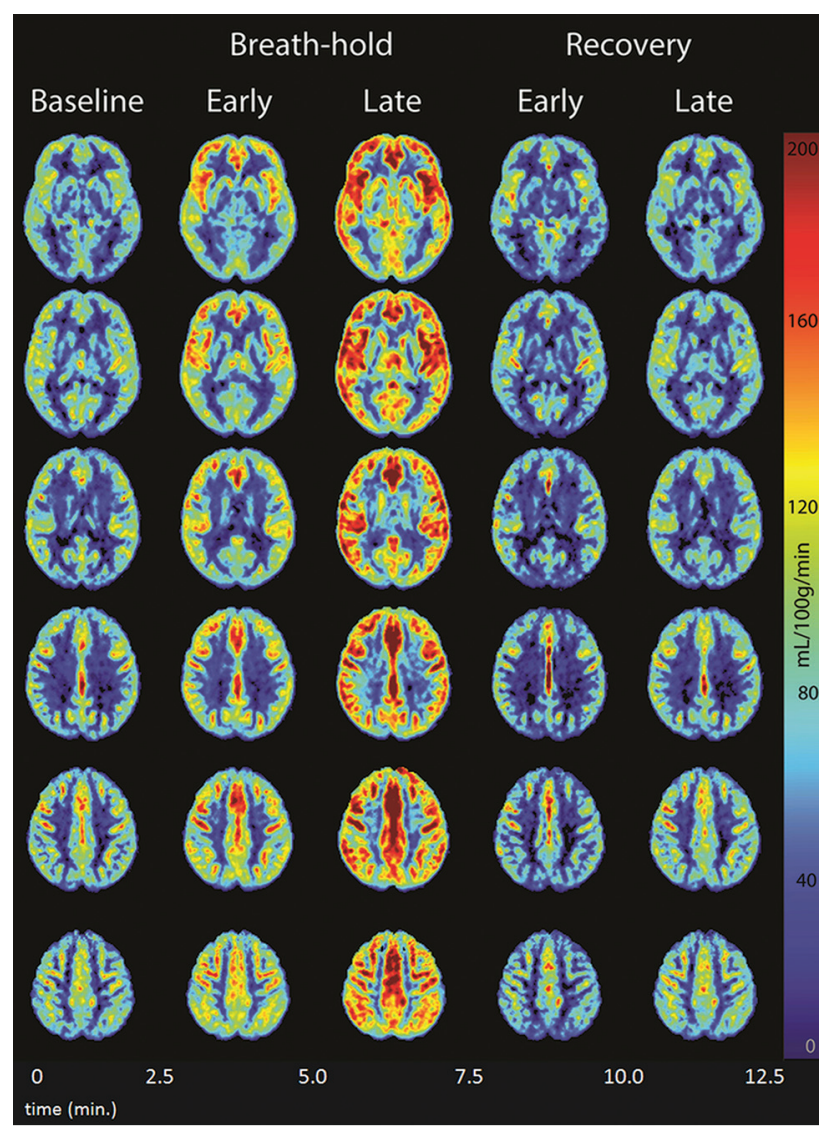

FIG 1. Mean CBF during the breath-hold experiment. False color maps of cohort mean cerebral blood flow during 5-minute breath-hold. Selected transverse brain sections of the cohort mean CBF before, during, and after the 5-minute breath-hold challenge. The mean total GM CBF over all participants and over all phases was scaled to 60 $\mathrm{mL} / 100 \mathrm{~g} / \mathrm{min}$. Likewise, the mean total WM CBF for all participants over all phases was scaled to $20 \mathrm{~mL} / 100 \mathrm{~g} / \mathrm{min}$ for WM.

rately by a paired $t$ test to identify differences between the anterior and posterior circulation. The influence of age and experience with prolonged breath-holds estimated from cumulative breathhold events of $>2$ minutes and 30 seconds was tested for correlation with CBF and ASL-sCoV using a mixed linear model with the participant as a random factor. Similarly, the relationships of the ASL parameters with $\mathrm{SpO}_{2}$ and $\mathrm{HR}$ were analyzed. Parametric testing occurred after testing for normal distribution. Generally, absolute CBF differences ( $\Delta$ in $\mathrm{mL} / 100 \mathrm{~g} / \mathrm{min}$ ) were used for descriptive statistics and statistical analyses, while ratios (in percentages) are mentioned for illustrative purposes.

\section{RESULTS}

\section{Cerebrovascular Reactivity}

All participants showed a significant CVR by an increase in CBF until 5 minutes of breath-hold with a subsequent decline at recovery $(P=.001$ for all vessel territories, GM, and WM; On-line Table and Figs 1 and $2 A,-B$ ). While the CBF increase from baseline was substantial after 5 minutes (late breath-hold scan phase for the total GM ROI: mean $\Delta \mathrm{CBF}, 18.3 \pm 14.4 \mathrm{~mL} / 100 \mathrm{~g} / \mathrm{min}$ $[+51.8 \%] ; P=.001)$, it was only subtle within the first $2.5 \mathrm{~min}$ utes of breath-hold (total GM ROI: mean $\triangle \mathrm{CBF}, 6.3 \pm 11.1 \mathrm{~mL}$ / $100 \mathrm{~g} / \mathrm{min}[+17.8 \%] ; P=.04)$. In 4 volunteers, early breath-hold- phase CBF was indeed lower than baseline CBF (mean, $-22.4 \%$; $P=.01)$. Baseline-to-late recovery phase CBF differences were not significant ( $P=.55$ for the total GM ROI). Return to baseline $\mathrm{CBF}$ or below occurred during the early recovery phase with a mild secondary CBF increase during the second 2 minutes and 30 seconds of recovery ( $P=.03$ in total GM ROI).

The absolute CBF and CVR of the anterior circulation (anterior cerebral artery, MCA) were, at all times, higher than in the posterior circulation $(P=.001$; On-line Table). The mean difference between CBF of the MCA and the posterior cerebral artery increased steadily during breath-hold from $8.8 \pm 6.6 \mathrm{~mL} / 100$ $\mathrm{g} / \mathrm{min}$ at baseline to $14.6 \pm 6.1 \mathrm{~mL} / 100 \mathrm{~g} / \mathrm{min}$ at late breath-hold $(P=.001)$.

During all scan phases, between-participant CBF variability showed a narrower range in the GM than in the WM as well as a lower overall CVR (CVR variability in GM: $83.8 \%-152.8 \%$ from baseline; CVR in WM: $74.2 \%-231.5 \%$ increase from baseline CBF; $P=.001$; On-line Table).

\section{Spatial Coefficient of Variation}

The ASL-sCoV varied over the course of the experiment $(P=$ $.001)$. In most cases $(n=12 / 15)$, there was an ASL-sCoV decrease between baseline and the early breath-hold phase (mean decrease: $-30.0 \% \pm 21.6 \% ; P=.002$; Fig $2 C)$. The ASL-sCoV remained reduced during the 5 -minute breath-hold ( $P=.81$ for the difference between early and late breath-hold) and rose again during recovery $(P=.01)$. There was no difference between the baseline and recovery phases $(P=.29)$.

The range of GM ASL-sCoV among participants was smaller during breath-hold than during normal breathing: mean, $2.8 \% \pm$ $1.0 \%$; range, $1.9 \%-5.3 \%$ versus mean, $1.8 \% \pm 0.4 \%$; range, $1.5 \%-3.0 \%$, for baseline versus early breath-hold phases, respectively. This range equates to a $35.7 \%$ decrease of ASL-sCoV variability among participants $(P=.02)$.

\section{Physiologic Correlations}

The dynamics of $\mathrm{SpO}_{2}$ and HR are presented in the On-line Table. $\mathrm{SpO}_{2}$ correlated with CVR, with an estimated increase of CBF of $0.82 \mathrm{~mL} / 100 \mathrm{~g} / \mathrm{min}$ with each $1 \% \mathrm{SpO}_{2}$ drop (95\% CI, 0.6-1.1 $\mathrm{mL} / 100 \mathrm{~g} / \mathrm{min} ; P=.001$; Fig $3 A,-B)$. HR was not correlated with CVR $(P=.36$; Fig $3 C,-D)$. Similarly, age was not identified as an influential cofactor on $\operatorname{CVR}(P=.32)$.

If one took the entire group of 15 freedivers into account, previous prolonged breath-hold events were not correlated with absolute CBF values $(P=.56)$. However, there were 2 outlier participants (divers 11 and 13) with exceptionally extensive experience and comparatively high CBF. After their exclusion, a relationship between previous breath-hold experience and absolute CBF was found with 1000 previous prolonged breath-holds, reducing CBF in GM by $2.2 \mathrm{~mL} / 100 \mathrm{~g} / \mathrm{min}$ (95\% CI, 0.7-3.7 mL/100 $\mathrm{g} / \mathrm{min}$; $P=.01$; Fig 4 ) for the remaining 13 cases. This finding was similar for $\mathrm{CBF}$ in WM: One thousand previous breath-holds reduced WM CBF by $0.6 \mathrm{~mL} / 100 \mathrm{~g} / \mathrm{min}$; 95\% CI, 0.15-1.1 mL/100 $\mathrm{g} / \mathrm{min}(P=.01)$. Mean CVR, however, as defined by the $\Delta \mathrm{CBF} /$ $\mathrm{CBF}_{0}$, was not correlated with previous experience with prolonged breath-holds $(P=.23)$. Similarly, ASL-sCoV was not cor- 

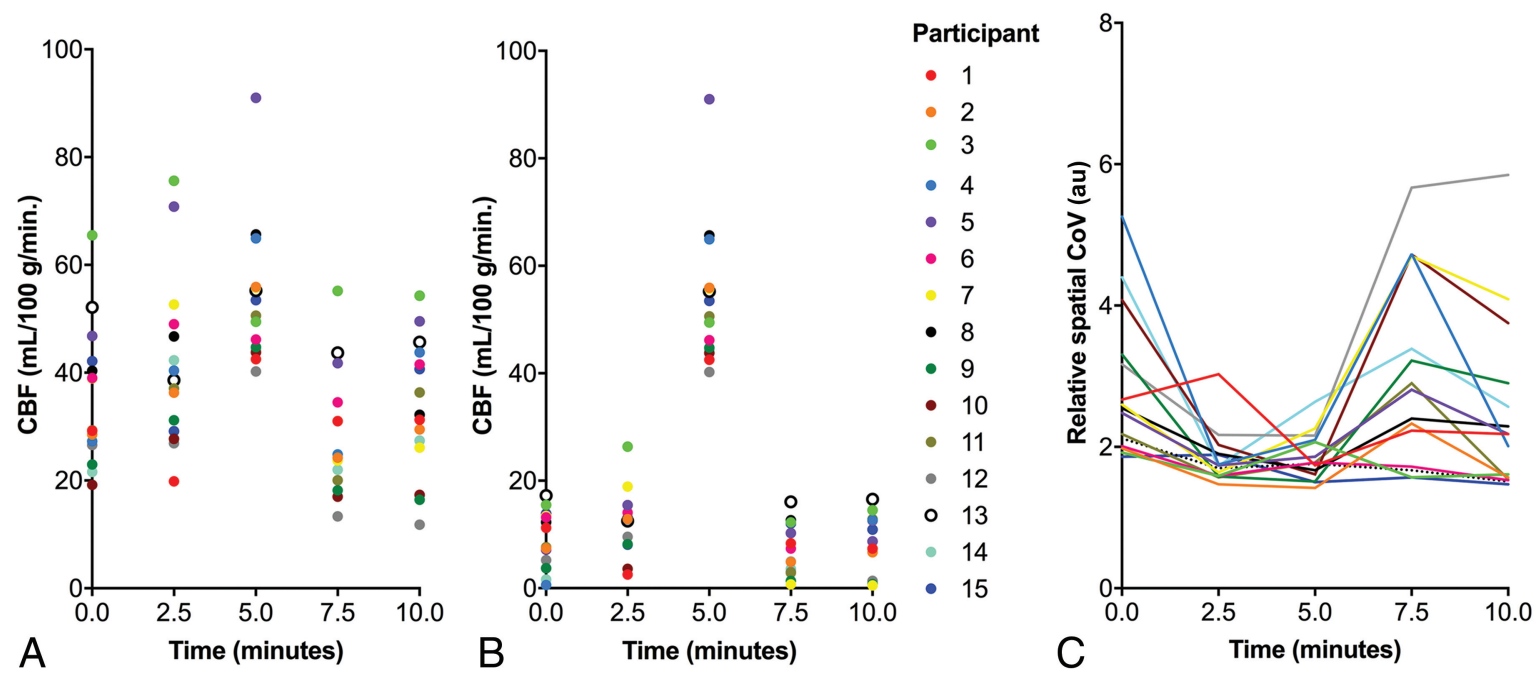

$-14$

B Time (minutes)

\section{C}

$-15$

FIG 2. Cerebral blood flow and partial volume-corrected relative spatial coefficient of variation dynamics. Baseline scan with normal ventilation starts at 0 minutes, the second and third measurement points represent 2 minutes and 30 seconds and 5 minutes of breath-hold, and the fourth and fifth scan phases at 7 minutes and 30 seconds and 10 minutes represent the recovery phase. $A$, CBF in gray matter voxels only ( $n=$ 15 participants, each represented by a color dot). B. The same for white matter only. C, Partial volume-corrected relative spatial coefficient of variation dynamics in gray matter. Y-axis: relative CBF spatial coefficient of variation defined as the ratio of the actual spatial coefficient of variation divided by the spatial coefficient of variation expected on the basis of anatomy (in arbitrary units [au]).

related with $\mathrm{SpO}_{2}, \mathrm{HR}$, age, or diver experience with prolonged breath-holds $(P=.45, .53, .90$, and .69 , respectively).

\section{MR Spectroscopy and Blood Analyses}

${ }^{31} \mathrm{P}-\mathrm{MR}$ spectroscopy (Fig 5A) revealed minor fluctuations in $\mathrm{pH}$ (Fig $5 B$ ) and ATP metabolites within physiologic ranges during the course of breath-hold $(P=.07$ for $\mathrm{pH} ; P>.05$ for dynamics in relative $\mathrm{PCr} ; \mathrm{P}_{\mathrm{i}}$; ATP- $\alpha,-\beta,-\gamma$; and phosphomono- and diester levels). There was a small-but-significant decrease of the PCr / (ATP + adenosine diphosphate) ratio between baseline and late-phase breath-hold in the matchedpairs analysis of the individuals $(-6.4 \% ; P=.02$; Fig $5 C)$. $\mathrm{PCr}$ and $\beta$-ATP differences relative to their baseline values $(\Delta \mathrm{PCr}$ and $\Delta \beta$-ATP) were correlated with the differences in $\mathrm{pH}$ from baseline $(R=0.53 ; P<.001$ for $\Delta \mathrm{PCr}$ and $R=0.45 ; P=.003$ for $\Delta \beta$-ATP, respectively; Fig $5 D)$.

${ }^{1} \mathrm{H}$-MR spectroscopy never showed the $\mathrm{CH}_{3}$ doublet of lactate at $1.34 \mathrm{ppm}$ in any of the participants. No significant changes in the levels of $N$-acetylaspartate, total creatine, or choline occurred $(P>.05$ for all $)$.

Venous blood samples showed a development of hypoxemia and hypercapnia during breath-hold (On-line Table, Fig 5E). A significant partial pressure of $\mathrm{CO}_{2}$ increase was noted only at late breath-hold $(P=.02)$, while $\mathrm{pO}_{2}$ had already dropped significantly after the early breath-hold phase $(P=.002)$. Both parameters returned to baseline after breath-hold, while venous lactate and glucose levels increased until the end of the experiment during the recovery phase $(P=.001$ and .01 , respectively; Fig $5 E)$.

\section{DISCUSSION}

This study provides 3 key findings. First, despite individual variability, freedivers show a relatively consistent and vessel-territoryspecific CVR during a breath-hold challenge, which is measurable with ASL. Second, CBF and the ATT correlate ASL-sCoV deliver independent aspects of the cerebrovascular response to breath- hold. Finally, this study identified indicators for an influence of earlier experience with prolonged breath-hold on absolute CBF during the breath-hold challenge but not on CVR itself. Physiologic responses can apparently withstand the extreme biochemical challenge induced by a prolonged breath-hold of 5 minutes, which can be detected by ASL and MR spectroscopy.

Multiple methods exist to assess CBF, including ASL, phasecontrast MR imaging, PET, and Doppler sonography. Maximum breath-hold studies are rare, and CBF evaluations were, until now, exclusively performed with Doppler sonography, which revealed a continuous elevation of flow velocity in the MCA of around $100 \% .{ }^{19,20}$ The mean maximum CBF increase after 5 minutes of breath-hold observed in this ASL study was 51.8\%, which is very close to values observed in ASL studies using hypercarbic gas inhalation or short breath-hold, but indeed in some cases lower than values measured with ASL in a recent maximum breath-hold study $(+107 \%){ }^{5,21,22}$ Previous studies comparing CBF assessments with different methods similarly revealed substantial intermethod differences in absolute CBF but otherwise confirmed a high correlation between methods. PET-estimated absolute CBF was, for example, consistently lower than phasecontrast MR imaging, while Doppler and ASL differed substantially in relative $\mathrm{CBF}$ change in a drug-stimulation trial. ${ }^{23,24}$ These differences are not surprising and can be explained by different influential factors acting on the respective flow parameters. While ASL can measure absolute CBF, Doppler provides flow velocity in a local vessel segment as a surrogate parameter for $\mathrm{CBF}$. $\mathrm{CO}_{2}$ is a strong vasodilating agent in cerebral tissue causing an increase in $\mathrm{CBF}$ due to increased blood volume (CBV) based on the equation $\mathrm{CBF}=\mathrm{CBV} / \mathrm{MTT}$. The blood flow velocity rises despite vessel dilation also due to reduced ATT. However, earlier Doppler studies revealed a strong neuromuscular response in proximal vessel segments of the anterior circulation, while more distal segments and the posterior circulation seemed less responsive. ${ }^{25,26}$ This 

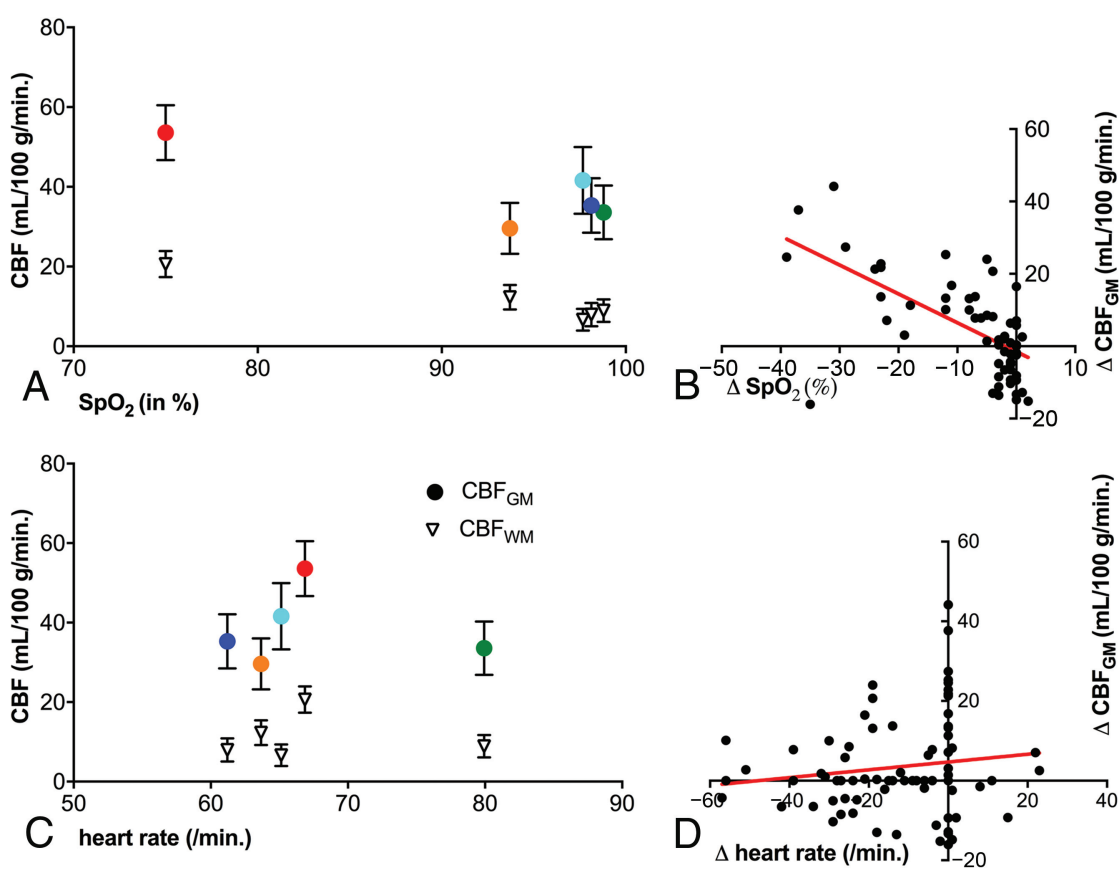

FIG 3. Relationship of $\mathrm{SpO}_{2}$ and heart rate with CBF. A, Mean GM CBF (colored dots) and mean WM CBF (triangles) are both strongly correlated with absolute $\mathrm{SpO}_{2}$. Color encoding for $A$ and $C$ : green for baseline, orange for 2 minutes and 30 seconds breath-hold, red for 5 -minute breathhold, turquoise after 2 minutes and 30 seconds of recovery, and blue after 5 minutes of recovery. The same WM time points are right below. $B, \triangle C B F$ (here measured in GM) similarly correlates well to $\Delta \mathrm{SpO}_{2}$ (all 75 single values). $C$ and $D$, There is no strong correlation between heart rate and CBF in absolute values (C, mean) or $\Delta \mathrm{CBF}$ and $\Delta \mathrm{SpO}_{2}(D)$.

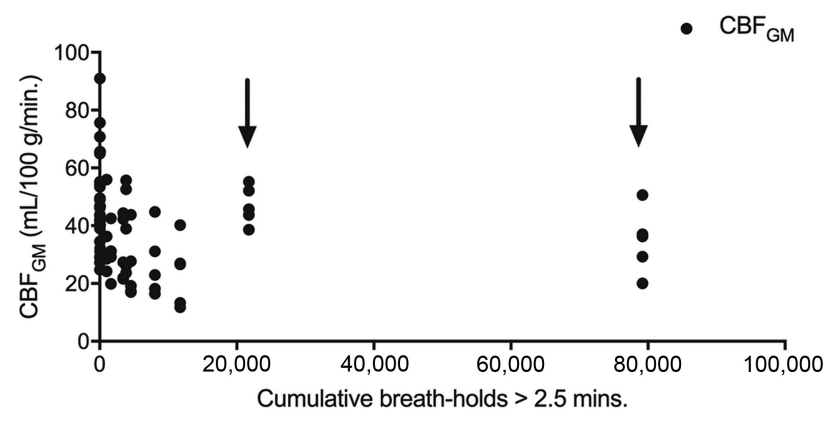

FIG 4. Scatterplot illustration of the relationship between cumulative breath-hold experience and CBF during the experiment. With the exception of 2 participants (divers 11 and 13, marked with arrows), there is a lower CBF during the experiment with more previous prolonged breath-hold events (cumulative breath-hold, $>2$ minutes and 30 seconds).

may explain why Doppler-assessed breath-hold experiments identify a higher absolute and relative CVR, mostly in the M1 segments of the MCA, than most of those applying ASL, which captures perfusion in most distal and nonmuscular vascular segments.

A heterogeneous CVR between vessels was also observed in the present ASL study with lower CBF and CVR in the posterior cerebral artery territories. This finding can be explained by differences in labeling efficiency between vascular territories as well as the longer ATT in the posterior vascular territory. Microanatomic differences leading to a diverse autoregulation capacity between the anterior and posterior circulation as an underlying reason for a diverse CVR are, on the other hand, more controversially discussed even beyond the field of perfusion studies under extreme conditions. ${ }^{27,28}$ A recent Doppler-monitored breath-hold study supports our findings of a lower CVR in the posterior cerebral artery and suggests a different sympathetic activation between the 2 territories as an additional explanation beyond the labeling aspect, which needs to be considered in ASL. ${ }^{29}$ An awareness of a vessel-selective CVR is, however, crucial when interpreting ASL measurements in focal ischemic lesions after prolonged clinical conditions of apnea.

Another finding of this study is that $\mathrm{CBF}$ decreased during the first 2 minutes and 30 seconds of breath-hold in 4 of 15 participants. The counter-suggestive relatively higher $\mathrm{CBF}$ at baseline compared with later time points might be the effect of anticipation anxiety toward the upcoming breath-hold challenge. ${ }^{30}$ This well-known mental phenomenon among freedivers is currently not sufficiently addressed in sports physiologic research. Indeed, our own results may only suggest that some freedivers experienced an early sympathetic activation before the breath-hold challenge. Due to the limited temporal resolution of the ASL sequence, which delivers a mean CBF over each of the 2 minutes and 30 seconds phases, we cannot readily assess how long the reduction of CBF persists and when exactly an elevation of the $\mathrm{CBF}$ above took place during the early breath-hold phase in these 4 volunteers.

We further confirmed a difference between the cortical GM and the deep WM CVR, with the total GM CBF known to be at least 2 times higher than the WM CBF. ${ }^{31}$ Low WM signal is an obstacle for WM CBF assessment in ASL, despite the availability of background suppression. ${ }^{31}$ However, CVR differences between GM and WM tissue can be interesting because they may help to better understand morphologic findings in brain diseases such as obstructive sleep apnea syndrome. ${ }^{32}$ We noted a larger CVR variability and relative increase in WM than in GM, which could be explained by the later arrival of blood in the relatively more distal WM vessels. WM CBF is difficult to measure at baseline due to longer ATT. Direct ATT assessment was technically not possible as part of our experimental setting due to temporal restrictions and a resulting mono-post-labeling delay ASL sequence. However, our ATT approximation based on ASL-sCoV confirmed a breath-hold-induced decrease in ATT, which might increase the SNR of WM CBF, which inflates the measured $\triangle \mathrm{CBF}$ to a certain extent.

For the interpretation of ASL-sCoV, it is viable to consider the methodologic peculiarities of ASL. An ATT increase will cause the ASL signal to appear in larger vessels, resulting in vascular artifacts. The ASL signal can, at the same time, decrease in areas with higher baseline ATT as in, for example, the perfusion watershed. These 2 effects both increase ASL-sCoV. ${ }^{8}$ However, ASL-sCoV 

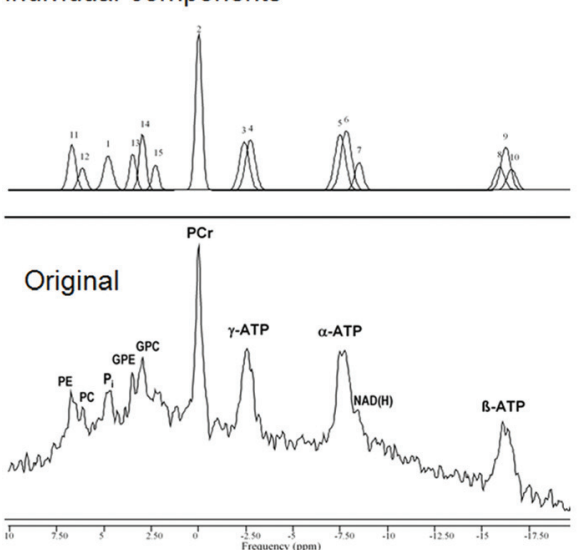

A

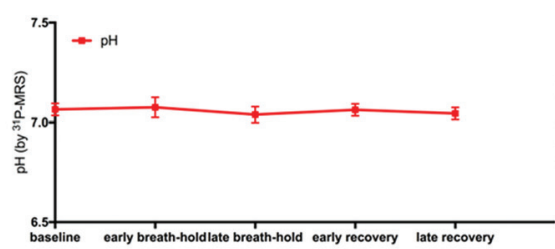

B

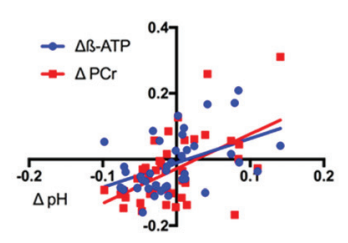

D

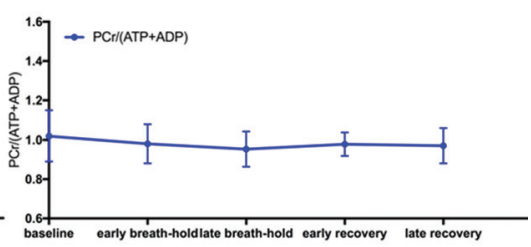

C

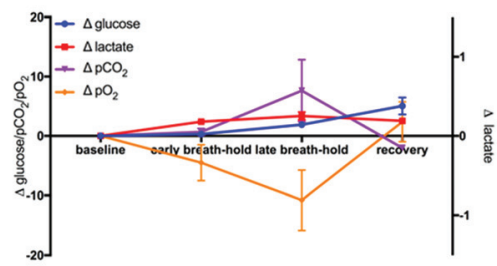

E

FIG 5. Energy metabolites and blood gases in brain and venous blood. A, Sample ${ }^{31}$ P-MR spectrum of 1 participant averaged over 2 minutes and 30 seconds of the late breath-hold phase (above), displayed together with AMARES-fitted spectral components (below). B, Brain pH as assessed by ${ }^{31} \mathrm{P}-\mathrm{MR}$ spectroscopy is near-constant during the entire breath-hold experiment. $C$, The PCr / (ATP + adenosine diphosphate) ratio measured by ${ }^{31} \mathrm{P}-\mathrm{MR}$ spectroscopy slightly decreases during breath-hold. $D, \mathrm{PCr}$ and $\beta$-ATP differences $(\Delta)$ from individual baseline values correlate with the $\mathrm{pH}$ differences from baseline, indicating that small tendencies toward acidosis and ATP depletion occur during breath-hold. $E$, Venous blood analyses reveal significant hypoxemia and hypercapnia development (left axis in millimeters of mercury) during breath-hold with fast recovery. Blood glucose (in milligrams/deciliter, left axis) and lactate (in mmol/L, right axis) rose during breath-hold and did not return to baseline. All values are expressed as differences from baseline.

has a theoretic lower limit attained when all labeled blood has arrived in the tissue and all vascular artifacts have already disappeared. Further ATT decrease beyond this limit will have only minimal effect on the ASL-sCoV. Reaching this lower limit of ASL-sCoV during the early breath-hold could explain the low ASL-sCoV variability in some participants. The ASL-sCoV decrease, however, appears to occur earlier than the CBF increase and levels off during the second breath-hold phase, while CBF further increases. An earlier response to breath-hold leading to decreasing ATT before the CBF increase in the late breath-hold phase can be the explanation, denoting that the effect is mainly vascular and perfusion changes as detected by ASL follow later. ASL-sCoV may be an earlier CVR marker of hypoxemia than absolute CBF.

CVR is predominantly triggered by changes in blood $\mathrm{CO}_{2}$, while, for example, hypoxemia detected by peripheral chemoreceptors is considered to play an independent-but-inferior role in cerebral vasodilation. ${ }^{33}$ While Willie et $\mathrm{al}^{20}$ already reported that $\mathrm{O}_{2}$ metabolism has a crucial influence on the breath-hold capability of freedivers, stressing the role of $\mathrm{O}_{2}$ for breath-hold tolerance and CVR, Cross et $\mathrm{al}^{19}$ could not confirm an influence of $\mathrm{O}_{2}$ on cerebral autoregulation in their prolonged breath-hold study. We identified falling $\mathrm{O}_{2}$ as an influential factor of CVR in this study, which can usually not be observed in CBF studies applying hypercarbic-normoxic gas despite otherwise comparable CVR between breath-hold and hypercarbic-normoxic gas studies. ${ }^{5,26}$ Due to the design of this study, which could not rely on arterial $\mathrm{CO}_{2}$ measurements, it remains, however, impossible to discern the relative contribution of hypercarbia and hypoxia to CBF increase. CVR increased faster in the second half of the breath-hold experiment in correlation with the secondarily faster $\mathrm{SpO}_{2}$ decline, which can be explained by pulmonary $\mathrm{O}_{2}$ stores that allow normal hemodynamic conditions during the first minutes of breath-hold. Cerebral near-infrared measurements in elite freedivers showed that cerebral $\mathrm{O}_{2}$ desaturation tends to occur within a mean of $175 \pm 50$ seconds, but not before, which supports our finding. ${ }^{34}$

In this study, CBF itself was not strongly correlated to HR. This finding does not allow concluding that CBF in breath-hold is not modulated by cardiac causes. It is, however, beyond the scope of this study to evaluate cardiac cofactors to CVR such as heart stroke volume or the diving reflex in detail. The factor age (an indirect measure of the cardiac and vascular influence on CVR) was assessed but was not associated with any of the flow parameters, which is not surprising considering that most volunteers in this study were younger than 40 years of age. Also, a selection bias of outstandingly healthy and well-trained freediver volunteers must be considered a further contributing factor.

A physiologic adaptation to breath-hold was another hypothesis to be tested in this study. A diminished CVR during hypercarbia/hypoxemia was reported for patients with chronic obstructive pulmonary disease as well as sleep apnea. ${ }^{35}$ In an attempt to investigate a similar association between experience with hypercarbic/hypoxemic states and CVR in freedivers, we estimated the total amount of previous prolonged breath-holds of the participants before participation in the current breath-hold experiment (defined as cumulative breath-holds of $>2$ minutes and $30 \mathrm{sec}$ onds in a lifetime). Our findings do not unconditionally corroborate that breath-hold experience accounts for an adaptation effect on CBF. First, CVR did not differ between more breathhold-experienced freedivers and their less experienced counterparts. Second, by far, the 2 most prolonged breath-hold-experienced freedivers, who additionally stated a high frequency of longer breath-holds per training session, showed relatively high CBF values. However, for the remaining cohort, an association between experience and lower absolute CBF in all phases of the experiment could be observed, which may indicate that repetitive previous hypercarbia and hypoxemia have an acute cerebrovas- 
cular effect during a breath-hold challenge. Due to the limited cohort size, which also included freedivers normally specialized in shorter breath-holds, this interesting and also clinically relevant observation will need to undergo further critical evaluation in the future, favorably in a more homogeneous group regarding age and freediving specialization.

In prolonged breath-hold, the efficacy of the cerebrovascular but also the cardiac response to maintain a stable $\mathrm{O}_{2}$ supply to the brain despite decreasing availability is a crucial health aspect and can be assessed spectroscopically regarding energy metabolism. Direct noninvasive in vivo measurements of brain energy metabolism during prolonged breath-hold are extremely rare. ${ }^{22,36}$ Cerebral lactate accumulation or acidosis was observed in none of our participants, suggesting a sufficient compensation of limited $\mathrm{O}_{2}$ supply by recruitment of ATP stores and increasing CBF. However, we identified a substantial decrease in the PCr / (ATP + adenosine diphosphate) ratio during breathhold. This can be interpreted as a compensatory PCr decrease to provide ATP by PCr hydrolysis as a consequence of declining $\mathrm{O}_{2}$ availability and reduced aerobic ATP production capacity in prolonged breath-hold.

Rising peripheral venous lactate levels during the breath-hold challenge in contrast to stable cerebral lactate stresses the shift toward a preferred cerebral $\mathrm{O}_{2}$ supply in breath-hold, including a $\mathrm{CBF}$ increase and, simultaneously, peripheral vasoconstriction. The rising glucose levels are likely a consequence of adrenalineinduced glucose mobilization and underline the exceptional metabolic and mental challenge of prolonged breath-hold. ${ }^{37}$ The correlative venous $\mathrm{pO}_{2}$ analyses documented pathologically low $\mathrm{O}_{2}$ levels after 5 minutes of breath-hold (down to $60 \% \mathrm{SpO}_{2}$ ) and corroborated similarities of this breath-hold experiment with clinical settings of hypoxemia. On the other hand, untrained persons may encounter life-threatening consequences under these circumstances, while freedivers face hypoxemia under voluntary and trained conditions.

Maximum breath-hold without contact with water is a particular challenge for freedivers because regular training sessions mostly include water immersion, which causes an augmented diving reflex. Only very few participants fulfilled the inclusion criteria and were able to perform sufficient breath-hold in the noisy MR imaging environment. Multiple candidates declined due to the lack of silence, or they would not volunteer for additional MR spectroscopy breath-hold experiments, thus the limited cohort size. Furthermore, participants did not tolerate a $\mathrm{CO}_{2}$ mask, which interfered with their meditative state. We therefore decided to analyze venous $\mathrm{CO}_{2}$. However, while venous $\mathrm{CO}_{2}$ can be used to confirm hypercapnia, it is, however, too variable to study the $\mathrm{CO}_{2}$ influence on CVR due to known substantial deviations from arterial $\mathrm{CO}_{2}$ in the brain. ${ }^{38}$ For this reason, we can also only assume that all normoventilating volunteers started at normal $\mathrm{CO}_{2}$ blood levels in the experiment. The unavailability of these otherwise valuable data limits the evaluation of the physiologic processes behind our observations.

\section{CONCLUSIONS}

This study revealed that experienced freedivers develop a CVR, which is sufficient to maintain a physiologic cerebral energy me- tabolism even during a prolonged breath-hold period of 5 minutes and severely diminishing blood $\mathrm{O}_{2}$. Furthermore, ASL parameters, which are determined by blood flow and vessel diameter alterations alike, serve as excellent candidate MR imaging parameters to reveal this response, while ${ }^{31} \mathrm{P}-\mathrm{MR}$ spectroscopy revealed its utility to dynamically study acute changes in cerebral energy metabolism. ASL may also provide evidence for long-term adaptation of cerebral vasculature following repetitive hypoxia-hypercapnia. Imaging and metabolic findings of the present freediver study can be used to better understand CVR during hypoxiahypercapnia in critical care and sleep apnea conditions.

\section{ACKNOWLEDGMENTS}

The authors acknowledge Dipl.-Phys. Jürgen Gieseke for his assistance with the ASL sequence implementation, and we thank all freedivers for their outstanding effort to make this study possible.

Disclosures: Burkhard Mädler—UNRELATED: Employment: Philips Healthcare, Comments: regular salary; Kim van de Ven—UNRELATED: Employment: Philips Healthcare. Comments: regular salary.

\section{REFERENCES}

1. AIDA, International Association for the Development of Apnea. Symbol of Freediving. SYMBhttps://www.aidainternational.org/ WorldRecords\#recordsMan. Accessed June 28, 2018

2. Li PA, Kristian T, Shamloo M, et al. Effects of preischemic hyperglycemia on brain damage incurred by rats subjected to 2.5 or 5 minutes of forebrain ischemia. Stroke 1996;27:1592-601, discussion 1601-02 Medline

3. Peppard PE, Young T, Barnet JH, et al. Increased prevalence of sleepdisordered breathing in adults. Am J Epidemiol 2013;177:1006-14 CrossRef Medline

4. Urback AL, MacIntosh BJ, Goldstein BI. Cerebrovascular reactivity measured by functional magnetic resonance imaging during breathhold challenge: a systematic review. Neurosci Biobehav Rev 2017;79: 27-47 CrossRef Medline

5. Tancredi FB, Hoge RD. Comparison of cerebral vascular reactivity measures obtained using breath-holding and $\mathrm{CO} 2$ inhalation. J Cereb Blood Flow Metab 2013;33:1066-74 CrossRef Medline

6. Pattinson KT, Governo RJ, MacIntosh BJ, et al. Opioids depress cortical centers responsible for the volitional control of respiration. J Neurosci 2009;29:8177-86 CrossRef Medline

7. Yadav SK, Kumar R, Macey PM, et al. Regional cerebral blood flow alterations in obstructive sleep apnea. Neurosci Lett 2013;555: 159-64 CrossRef Medline

8. Mutsaerts HJ, Petr J, Václavů L, et al. The spatial coefficient of variation in arterial spin labeling cerebral blood flow images. J Cereb Blood Flow Metab 2017;37:3184-92 CrossRef Medline

9. Erecińska M, Silver IA. ATP and brain function. J Cereb Blood Flow Metab 1989;9:2-19 CrossRef Medline

10. Clement P, Mutsaerts HJ, Vaclavu L, et al. Variability of physiological brain perfusion in healthy subjects: a systematic review of modifiers: considerations for multi-center ASL studies. J Cereb Blood Flow Metab 2017 Jan 1:271678X17702156. [Epub ahead of print] CrossRef Medline

11. Mutsaerts HJ, Lysvik E, Schrantee A, et al. ExploreASL: image processing toolbox for multi-center arterial spin labeling population analyses. In: Proceedings of the Congress and General Assembly of the European Society of Magnetic Resonance in Medicine and Biology, Barcelona, Spain. October 19-21, 2017

12. Ashburner J. A fast diffeomorphic image registration algorithm. Neuroimage 2007;38:95-113 CrossRef Medline

13. Alsop DC, Detre JA, Golay X, et al. Recommended implementation of arterial spin-labeled perfusion MRI for clinical applications: a consensus of the ISMRM perfusion study group and the European 
consortium for ASL in dementia. Magn Reson Med 2015;73:102-16 CrossRef Medline

14. Tatu L, Moulin T, Bogousslavsky J, et al. Arterial territories of the human brain: cerebral hemispheres. Neurology 1998;50:1699-708 CrossRef Medline

15. Asllani I, Borogovac A, Brown TR. Regression algorithm correcting for partial volume effects in arterial spin labeling MRI. Magn Reson Med 2008;60:1362-71 CrossRef Medline

16. Naressi A, Couturier C, Devos JM, et al. Java-based graphical user interface for the MRUI quantitation package. MAGMA 2001;12: 141-52 Medline

17. McCanny P, Bennett K, Staunton P, et al. Venous vs arterial blood gases in the assessment of patients presenting with an exacerbation of chronic obstructive pulmonary disease. Am J Emerg Med 2012;30: 896-900 CrossRef Medline

18. Vickers AJ. The use of percentage change from baseline as an outcome in a controlled trial is statistically inefficient: a simulation study. BMC Med Res Methodol 2001;1:6 CrossRef Medline

19. Cross TJ, Kavanagh JJ, Breskovic T, et al. Dynamic cerebral autoregulation is acutely impaired during maximal apnoea in trained divers. PLoS One 2014;9:e87598 CrossRef Medline

20. Willie CK, Ainslie PN, Drvis I, et al. Regulation of brain blood flow and oxygen delivery in elite breath-hold divers. J Cereb Blood Flow Metab 2015;35:66-73 CrossRef Medline

21. Zhou Y, Rodgers ZB, Kuo AH. Cerebrovascular reactivity measured with arterial spin labeling and blood oxygen level dependent techniques. Magn Reson Imaging 2015;33:566-76 CrossRef Medline

22. Vestergaard MB, Larsson HB. Cerebral metabolism and vascular reactivity during breath-hold and hypoxic challenge in freedivers and healthy controls. J Cereb Blood Flow Metab 2017 Jan 1:271678X17737909. [Epub ahead of print] CrossRef Medline

23. Sorond FA, Hollenberg NK, Panych LP, et al. Brain blood flow and velocity: correlations between magnetic resonance imaging and transcranial Doppler sonography. J Ultrasound Med 2010;29: 1017-22 CrossRef Medline

24. Vestergaard MB, Lindberg U, Aachmann-Andersen NJ, et al. Comparison of global cerebral blood flow measured by phase-contrast mapping MRI with (15) O-H2 O positron emission tomography. J Magn Reson Imaging 2017;45:692-99 CrossRef Medline

25. Sato K, Sadamoto T, Hirasawa A, et al. Differential blood flow responses to $\mathrm{CO}(2)$ in human internal and external carotid and vertebral arteries. J Physiol 2012;590:3277-90 CrossRef Medline

26. Villien M, Bouzat P, Rupp T, et al. Changes in cerebral blood flow and vasoreactivity to $\mathrm{CO} 2$ measured by arterial spin labeling after 6 days at 4350m. Neuroimage 2013;72:272-79 CrossRef Medline

27. Nakagawa K, Serrador JM, Larose SL, et al. Autoregulation in the posterior circulation is altered by the metabolic state of the visual cortex. Stroke 2009;40:2062-67 CrossRef Medline

28. Flück D, Beaudin AE, Steinback CD, et al. Effects of aging on the association between cerebrovascular responses to visual stimulation, hypercapnia and arterial stiffness. Front Physiol 2014;5:49 CrossRef Medline

29. Bruce CD, Steinback CD, Chauhan UV, et al. Quantifying cerebrovascular reactivity in anterior and posterior cerebral circulations during voluntary breath holding. Exp Physiol 2016;101:1517-27 CrossRef Medline

30. Hasler G, Fromm S, Alvarez RP, et al. Cerebral blood flow in immediate and sustained anxiety. $J$ Neurosci 2007;27:6313-19 CrossRef Medline

31. van Osch MJ, Teeuwisse WM, van Walderveen MA, et al. Can arterial spin labeling detect white matter perfusion signal? Magn Reson Med 2009;62:165-73 CrossRef Medline

32. Chen HL, Lin HC, Lu CH, et al. Systemic inflammation and alterations to cerebral blood flow in obstructive sleep apnea. J Sleep Res 2017;26:789-98 CrossRef Medline

33. Ogoh $\mathrm{S}$, Nakahara $\mathrm{H}$, Ueda $\mathrm{S}$, et al. Effects of acute hypoxia on cerebrovascular responses to carbon dioxide. Exp Physiol 2014;99: 849-58 CrossRef Medline

34. Eichhorn L, Erdfelder F, Kessler F, et al. Evaluation of near-infrared spectroscopy under apnea-dependent hypoxia in humans. J Clin Monit Comput 2015;29:749-57 CrossRef Medline

35. Beaudin AE, Hartmann SE, Pun M, et al. Human cerebral blood flow control during hypoxia: focus on chronic pulmonary obstructive disease and obstructive sleep apnea. J Appl Physiol (1985) 2017;123: 1350-61 CrossRef Medline

36. Bainbridge A, Tachtsidis I, Faulkner SD, et al. Brain mitochondrial oxidative metabolism during and after cerebral hypoxia-ischemia studied by simultaneous phosphorus magnetic-resonance and broadband near-infrared spectroscopy. Neuroimage 2014;102(Pt 1): 173-83 CrossRef Medline

37. Eichhorn L, Erdfelder F, Kessler F, et al. Influence of apnea-induced hypoxia on catecholamine release and cardiovascular dynamics. Int J Sports Med 2017;38:85-91 CrossRef Medline

38. Kelly AM. Review article: can venous blood gas analysis replace arterial in emergency medical care. Emerg Med Australas 2010;22: 493-98 CrossRef Medline 